\title{
Corona-Pandemie: eine besondere Herausforderung in Geriatrie und Gerontopsychiatrie
}

Das BGPN-Mitglied PD Dr. Eva Brandl beschäftigt sich mit psychischen Erkrankungen über die Lebensspanne. Bei der Frühjahrstagung der BGPN 2019 hat sie über Psychopharmaka in der Schwangerschaft vorgetragen, heute berichtet sie über ihre Erfahrungen als Oberärztin des gerontopsychiatrischen Bereichs der Psychiatrischen Universitätsklinik der Charité im St. Hedwig-Krankenhaus. Weitere Interessenschwerpunkte sind postpartale Erkrankungen, Polypharmazie, Pharmakogenetik und transkulturelle Psychiatrie.

Die Corona-Pandemie stellt uns vor nie dagewesene Herausforderungen. Ältere Menschen sind in besonderem Maße von den Einschränkungen betroffen. Hier stellt für die meisten Patienten weniger Covid-19 ein Problem dar als die Kontaktbeschränkungen zur Eindämmung der Pandemie. Aufgrund des hohen Risikos für an Covid-19-erkrankte ältere Menschen werden auf vielen geriatrischen und gerontopsychiatrischen Stationen besonders strenge Schutzvorschriften eingehalten. Die Einschränkungen bis zur völligen Einstellung von Besuchen sind gerade bei längeren Krankenhausaufenthalten eine erhebliche Belastung. Telefonkontakte zu Familie und Freunden sind häufig aufgrund von Hörminderung nur eingeschränkt nutzbar. Mangelnde Erfahrung und Berührungsängste verhindern die Nutzung von Apps und Videosystemen zur Interaktion. Für Menschen mit Demenz oder anderen kognitiven Einschränkungen sind die Schutzmaßnahmen schwer zu verstehen, was die Belastung deutlich erhöht. Fehlender persönlicher Kontakt zu ihren Familien ist für viele eine deutlich größere Belastung als die Vorstellung, an Covid-19 zu erkranken.

Über die Besuchseinschränkungen hinaus gibt es weitere erschwerende Veränderungen im Behandlungssetting. So haben viele Kliniken ihr elektives Aufnahmeprogramm reduziert oder eingestellt, sodass der $\mathrm{Zu}$ - gang zur Behandlung komplizierter ist als sonst. Die Kommunikation mit dem Klinikpersonal ist durch den Mund-Nase-Schutz, gerade für Menschen mit Hörminderung, erschwert. Personal wird dadurch teilweise schlechter wiedererkannt, was den Beziehungsaufbau beeinträchtigen kann. Die Abstandsregelungen und Beschränkungen von Gruppenangeboten führen dazu, dass sich viele Patienten im Krankenhaus einsam fühlen.

Auch außerhalb des psychiatrischen Behandlungssettings tragen die mit der Pandemie verbundenen Maßnahmen zur Belastung älterer Personen bei. Viele Menschen haben im häuslichen Umfeld wenig oder gar keine persönlichen Kontakte zu ihren Angehörigen. Reduktion oder völlige Einstellung von Tagesstätten, Seniorentreffpunkten und aufsuchenden Angeboten sind ebenso problematisch wie der Wegfall von anderen kulturellen und gesellschaftlichen Möglichkeiten, den Alltag aktiv zu gestalten, z. B. Gottesdienst- oder Konzertbesuche.

Nicht zuletzt erhöhen die zu Beginn der Pandemie verbreitete Wahrnehmung, dass Covid-19 vorwiegend eine Gefahr für ältere Personen sei sowie die anfänglich geführten Diskussionen um Triage für die verfügbaren Intensivkapazitäten das Risiko für eine erhebliche Altersdiskriminierung. Fälle unzureichender Dokumentationen von Covid-Todesfällen unter Heimbewohnern, teilweise unzureichende oder überschießende Sicherheitsmaßnahmen für ältere Menschen und Rufe nach einer besonders strengen Isolierung von Menschen höheren Alters sind verschiedene Facetten dieses Problems [1]. Natürlich gibt es auch positive Gegenbeispiele, u. a. die sehr schnell entstandenen Nachbarschaftshilfenetzwerke. Dennoch birgt die Wahrnehmung von Covid-19 als „Alte-Leute-Problem “ gesellschaftliche Risiken, die sich auch in der psychischen Belastung dieser Altersgruppe widerspiegeln.

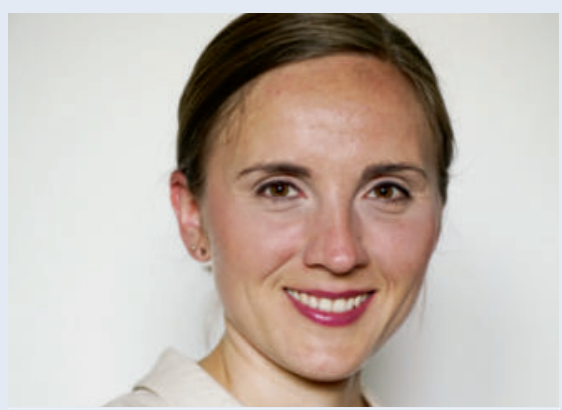

Priv.-Doz. Dr. Eva Brandl, Quelle: @privat

Ein Ruf nach schneller Beendigung der Pandemiemaßnahmen wäre zu kurz gegriffen. Eine verstärkte Wahrnehmung der besonderen psychischen Belastung und Isolation älterer Personen sowohl während einer Krankenhausbehandlung als auch im häuslichen Umfeld und eine Berücksichtigung dieser Faktoren in der Diskussion um die vermutlich längerfristig erforderlichen Schutzmaßnahmen wäre jedoch wünschenswert.

\section{Priv.-Doz. Dr. Eva Brandl, Berlin}

Literatur

[1] Fraser S, et al. Age Ageing 2020 May 6. epub ahead of print

IMPRESSUM

Prof. Dr. Tom Bschor

Redaktion: Dr. Anja M. Bauer

Berliner Gesellschaft für Psychiatrie und Neurologie e. V.

Schlosspark-Klinik, Abteilung für

Psychiatrie

Heubnerweg 2, 14059 Berlin

info@bgpn.de,www.bgpn.de 